\title{
Tumor Less Than or Equal to 2.0 Centimeters
}

National Cancer Institute

\section{Source}

National Cancer Institute. Tumor Less Than or Equal to 2.0 Centimeters. NCI Thesaurus.

Code C120285.

A tumor where the greatest dimension is less than or equal to 2.0 centimeters. 